\title{
Atributos Físico-hídricos de um Cambissolo Húmico Sob Sistema Agroflorestal no Planalto Catarinense
}

\author{
Jânio dos Santos Barbosa ${ }^{1}$, Kristem do Carmo Rosa Silva ${ }^{1}$, \\ Carla Eloize Carducci ${ }^{2}$, Karine Louise dos Santos ${ }^{1}$, Letícia Salvi Kohn ${ }^{1}$, \\ Jonathan Santos Fucks ${ }^{1}$ \\ ${ }^{1}$ Departamento de Agricultura, Biodiversidade e Florestas, \\ Universidade Federal de Santa Catarina - UFSC, Curitibanos/SC, Brasil \\ ${ }^{2}$ Faculdade de Ciências Agrárias, Universidade Federal da Grande Dourados - UFGD, Dourados/MS, Brasil

\begin{abstract}
RESUMO
Objetivou-se com este trabalho avaliar e comparar a viabilidade de um sistema agroflorestal a partir dos atributos físicos e hídricos do solo ao longo do tempo, conduzido na cidade de Curitibanos-SC com tratamentos: SAF-Erva Mate (SE), SAF-Frutífera (SF), SAF-Agrícola (SA), e uma área de Vegetação Nativa (VN) como testemunha. Foram abertas trincheiras aleatórias em cada tratamento para coleta das amostras de solo com estrutura preservada e alterada nas profundidades 0,0-0,05 $\mathrm{m}$ e 0,05-0,20 m no primeiro semestre de $2014 \mathrm{e} 2015$. As profundidades foram selecionadas de acordo com análises visuais da estrutura do solo. Foram determinados os atributos físicos e hídricos do solo e o balanço hídrico. A densidade do solo e a microporosidade proporcionaram um aumento significativo de seus atributos no decorrer do tempo. O sistema manteve-se dentro da capacidade de água disponível com balanço hídrico adequado ao desenvolvimento das culturas, o que o torna viável e sustentável.
\end{abstract}

Palavras-chave: qualidade estrutural, água no solo, balanço hídrico.

\section{Physical-hydric Attributes of a Humic Inceptisol in Agroforestry on the Santa Catarina Plateau}

\begin{abstract}
The present study has the objective to evaluate and compare the feasibility of agroforestry systems by physical and hydric soil attributes over time, conducted in the city of Curitibanos-SC. The treatments are as follows: SIF-mate herb (SE), SIF-fruit (SF), SIF-agricultural (SA) and Native Vegetation (NV) as a control area. Random trenches were dug in each treatment to collect soil samples with preserved and altered structure at $0.0-0.05 \mathrm{~m}$ and $0.05-0.20 \mathrm{~m}$ depths in the first half of 2014 and 2015, to determine physical and hydric soil attributes and the water balance. The depths were selected based on visual analysis of soil structure. Soil bulk density and microporosity provided a significant increase in its attributes over the course of time. The system remained within the available water capacity with proper water balance for the cultures, making it viable and sustainable.
\end{abstract}

Keywords: structural quality, soil water, water balance. 


\section{INTRODUÇÃO}

Os sistemas agroflorestais (SAFs) são uma alternativa de produção agrícola viável à agricultura familiar, por promover melhorias na qualidade física do solo devido à redução de efeitos negativos em sua estrutura, provocados por sistemas de manejo convencionais. Esse sistema integra várias espécies agrícolas com arbóreas cultivadas intercaladas e/ou animais domésticos, na mesma unidade de manejo, ou alternadas no tempo e no espaço (Silva et al., 2011). As espécies geralmente utilizadas apresentam diferentes ciclos, portes e funções que proporcionam ao ambiente edáfico um aumento na biodiversidade, o que promove melhorias físico-químicas, hídricas e microbiológicas do solo (Silva et al., 2011).

A redução de impactos negativos ao solo pelo o uso dos SAFs, especialmente em solos com baixo potencial para o cultivo, como é o caso da maioria dos Cambissolos, permite sua incorporação ao processo produtivo (Severiano et al., 2009). Esse sistema de manejo conservacionista tem originado discussões positivas quanto à melhoria na qualidade estrutural do solo; de acordo com Pezarico et al. (2013), o uso de práticas que minimizam as alterações na estrutura do solo promovem um sistema físico-hídrico favorável à infiltração, retenção e disponibilidade de água, incremento em carbono orgânico do solo de diferentes tipos e composições, além de facilitar o crescimento e o desenvolvimento do sistema radicular das plantas.

As alterações na estrutura do solo promovidas pelos diferentes sistemas de manejo podem ser visualizadas pelo método do Perfil Cultural diretamente no campo (Tavares et al., 1999). Esse método tem por finalidade destacar diferenças morfológicas no perfil, com vistas ao entendimento da ação antrópica na alteração da estrutura do solo e desta no desenvolvimento radicular, na dinâmica da água e transporte de nutrientes (Tavares et al., 1999; Ball et al., 2007; Giarola et al., 2010), além de prever o nível de compactação (Reichert et al., 2010).

Dessa forma, o grau de alteração da estrutura do solo detectado a campo ainda está vinculado às mudanças climáticas (regimes de chuvas) e à prática escolhida para manejo. Esses fatores facilitam o entendimento sobre a dinâmica da água no sistema solo-planta em agroflorestas e se faz necessário devido ao uso eficiente da água armazenada no solo, que geralmente está em maiores profundidades (Serafim et al., 2013). Sendo de suma importância conhecer os procedimentos que contribuem para uma produção sustentável, ou seja, compatíveis com a manutenção e recuperação do meio ambiente e a produção de alimentos (Loss et al., 2009). Assim, o presente trabalho tem como objetivo avaliar e comparar a viabilidade de um sistema agroflorestal a partir dos atributos físicos e hídricos do solo ao longo do tempo.

\section{MATERIAL E MÉTODOS}

\subsection{Descrição da área experimental}

O estudo foi conduzido na área experimental agroflorestal com 0,80 ha, localizada na Universidade Federal de Santa Catarina na cidade de Curitibanos, SC, e implantado em outubro de 2013. O clima da região é do tipo subtropical úmido mesotérmico (Cfb) segundo a classificação de Köppen (Wrege et al., 2011).

O sistema agroflorestal está constituído em uma área onde anteriormente houve cultivo de pinus, o qual foi removido no ano de 2010. No ano de 2013, foram utilizadas algumas espécies nativas já presentes na área, a exemplo de espécies florestais como Araucaria Angustifolia (Pinheiro-do-Paraná), Mimosa scabrella (Bracatinga), Solanum mauritianum (fumo bravo), Lithraea molleoides (bugreiro), Matayba eleagnoides (camboatá branco), canelas e aroeiras, entre outras.

Gradativamente as áreas foram organizadas em parcelas sendo essas enriquecidas por espécies de interesse, compondo os seguintes tratamentos: SAF-Erva Mate (SE) (Ilex paraguariensis), SAF-Frutíferas (SF), utilizando a pitangueira (Eugenia uniflora L.), a goiaba serrana (Acca sellowiana), araça (Psidium cattlelyanum), ingá (Inga spp), cerejeira (Eugenia involucrata), guabiroba (Campomanesia xanthocarpa), SAF-Agrícola (SA), com rotações de culturas: milho (Zea Maiz) e mandioca (Manihot esculenta) no verão e espécies de cobertura como azevém, aveia e trevo durante o inverno.

Todas as atividades de manejo do SAF evitam o revolvimento excessivo do solo, não utilizando defensivos agrícolas convencionais ou fertilizantes de alta solubilidade. A correção do solo é realizada através de calagem e aplicação de cinza, fosfato natural e composto orgânico. O único tratamento fitossanitário 
realizado é o controle de formigas com o uso de iscas à base de óleo de Neen.

O experimento consta ainda de uma área de proteção ambiental e foi utilizada como área testemunha Vegetação Nativa (VN).

Estes tratamentos estão distribuídos em parcelas com dimensões $500 \mathrm{~m}^{2}$, sendo que cada tratamento apresenta três repetições.

O solo sob o sistema agroflorestal foi classificado de acordo com a EMBRAPA (2011) como Cambissolo Húmico Alumínico muito argiloso (Tabela 1).

\subsection{Amostragem e Análise visual da estrutura do solo}

Foram abertas três trincheiras aleatórias em cada tratamento, para avaliação do perfil cultural (Tavares et al., 1999). Por meio dessa técnica, obtiveram-se os critérios de descrição dos modos de organização do solo, sendo utilizadas as seguintes simbologias: Nível 1 - AM = Volume de solo visualmente alterado pelo manejo, isto é, pelo maquinário e pelas raízes; $\mathrm{NAM}=$ Volume de solo visualmente não alterado pelo manejo; L=Volume de solo livre, solto, constituído por terra fina, agregados e torrões de tamanhos variados (de 0 a $10 \mathrm{~cm}$ ) sem nenhuma coesão; Nível 2 - do estado interno dos torrões presentes nos diferentes modos de organização do volume de solo antropizado: $\mu=$ Agregado não compactado; $\Delta=$ Agregado compactado; $\mu \Delta / \Delta \mu=$ Agregado em algum estádio de compactação, ou seja, a) $\mu \Delta=$ agregados que estão em processo de compactação, mas que ainda guardam predominantemente as características do estado não compacto $(\mu)$ sobre as características do estado compacto ( $\Delta)$; b) $\Delta \mu=$ agregados que estão fortemente compactos.
Conjuntamente à análise do perfil cultural, foi utilizada a análise visual da estrutura (VESS) para identificação da qualidade estrutural do solo proposta por Ball et al. (2007), na qual se pontua a qualidade do agregado por meio de notas como: Qe1 = Friável (estrutura boa); Qe2 = Intacto; Qe3 = Firme; Qe4 Compacto; Qe5 = Muito compacto (estrutura pobre). Dessa forma, definem-se cinco escores visuais (Ev) para a classificação da qualidade: $\mathrm{de} \mathrm{Ev}=1$ (melhor qualidade estrutural) a $\mathrm{Ev}=5$ (pior qualidade estrutural).

Através das análises visuais, identificaram-se duas camadas de solo com estrutura contrastante a $0,0-0,05 \mathrm{~m}$ e $0,05-0,20 \mathrm{~m}$, nas quais foram coletadas amostras de solo com estrutura preservada em anéis volumétricos com dimensão de $0,06 \mathrm{~m}$ de diâmetro e $0,025 \mathrm{~m}$ de altura e amostras com estrutura alterada, ambas em triplicata no primeiro semestre de 2014 e 2015.

\subsection{Análises físicas e hídricas do solo}

A partir das amostras alteradas, foram determinadas a granulometria pelo método da pipeta e o conteúdo de carbono orgânico do solo (EMBRAPA, 2011). Os valores obtidos de carbono orgânico foram transformados em matéria orgânica de acordo com a equação: $\mathrm{Mo}=\mathrm{C}^{*} 1,724$, onde: $\mathrm{Mo}=$ matéria orgânica $\left(\mathrm{g} \mathrm{kg}^{-1}\right)$; $\mathrm{C}=$ é o carbono orgânico $\left(\mathrm{g} \mathrm{kg}^{-1}\right)$ e 1,724 é um fator utilizado em virtude de se admitir que, na composição média do húmus, o carbono participa com $58 \%$.

Nas amostras com estrutura preservada, foram determinadas a densidade do solo (Ds), a porosidade total (PT) pelo método do anel saturado, a microporosidade (MI) (valor referente a $-6 \mathrm{kPa}$ ) e a macroporosidade (Ma) (EMBRAPA, 2011).

Tabela 1. Caracterização granulométrica do Cambissolo Húmico em diferentes profundidades em sistema agroflorestal no Planalto Catarinense.

Table 1. Particle size characterization of Inceptisol at different depths in agroforestry system in Santa Catarina Plateau.

\begin{tabular}{|c|c|c|c|c|c|c|}
\hline \multirow{3}{*}{ Tratamentos } & \multicolumn{3}{|c|}{$0-0,05 \mathrm{~m}$} & \multicolumn{3}{|c|}{$0,05-0,20 \mathrm{~m}$} \\
\hline & Argila & Areia & Silte & Argila & Areia & Silte \\
\hline & ....... & ........ & ... & & & \\
\hline $\mathrm{VN}$ & 598 & 91 & 311 & 645 & 88 & 267 \\
\hline SA & 648 & 61 & 291 & 623 & 62 & 315 \\
\hline SF & 655 & 67 & 278 & 640 & 58 & 302 \\
\hline SE & 656 & 76 & 268 & 578 & 78 & 345 \\
\hline
\end{tabular}

SE: SAF-Erva Mate; SA: SAF-Agrícola; SF: SAF-Frutífera e VN: Vegetação Nativa. 
Para determinação da capacidade de campo (CC, $-6 \mathrm{kPa}$ ) e ponto de murcha permanente (PMP, $-1500 \mathrm{kPa}$ ), foram utilizadas 32 amostras referências, encaminhadas ao Laboratório de Física do Solo - UFLA, onde, após saturação gradual, as amostras foram submetidas à unidade de sucção e às câmaras de pressão de placa porosa. A partir das amostras referências, foi realizado o controle de umidade nas demais (16 amostras), na UFSC - Campus de Curitibanos. Posteriormente, foram determinados a capacidade de água disponível do Cambissolo em estudo $(\mathrm{CAD}=6 \mathrm{kPa}-1500 \mathrm{kPa}$ * $\mathrm{Z}$ [ conteúdo de água a - 6 e a $-1500 \mathrm{kPa}, \mathrm{Z}$ : profundidade]) e o armazenamento de água no solo $\left(\sum_{i=1}^{n} \theta i\right)$ de acordo com (Reichardt \& Timm, 2004).

Para o balanço hídrico climatológico, utilizou-se a metodologia Thornthwaite \& Mather (1955), elaborado para determinar o regime hídrico de um local, sendo definida pela capacidade de água disponível (CAD) no solo, os valores de precipitação $(\mathrm{P})$ e evapotranspiração de referência (ETo), para o período de março/2014 a maio/2015. Os dados climatológicos foram adquiridos da estação meteorológica instalada na UFSC-Campus de Curitibanos.

\subsection{Análise estatística}

O delineamento utilizado foi em blocos ao acaso, em esquema de parcelas subsubdivididas, sendo a parcela o tempo: ano de 2014 e ano de 2015, a subparcela: os tratamentos (SAF-Erva Mate, SAF-Frutíferas, SAF-Agrícola e VN-Vegetação Nativa), e a subsubparcelas: as profundidades (0-0,05 $\mathrm{m}$ e 0,05-0,20 m). Os dados foram submetidos ao teste de normalidade de Shapiro-Wilk e, posteriormente à análise de variância, quando significativo, foi realizado o teste de comparação de médias de Tukey $(\mathrm{p}<0,05)$.

\section{RESULTADOS E DISCUSSÃO}

\subsection{Análise visual da estrutura do solo}

A avaliação da estrutura apoiou-se na aparência, resistência e nas características das unidades estruturais do solo ou camadas. Assim, foi possível identificar se em cada tratamento houve ao menos duas camadas de solo com diferenças na organização estrutural (Tabela 2).

Os perfis apresentaram modificações visíveis na organização dos agregados, com diferenciação entre os volumes antropizados, ou seja, os valores dos escores visuais (EV) variaram de 1,83 a 2,30 para a vegetação nativa $(\mathrm{VN})$ e SAF-Agrícola (SA), respectivamente. No entanto, as unidades estruturais apresentaram-se mais soltas $(\mathrm{L})$ com tendência à compactação dos agregados no SAF-Agrícola (SA) na camada superficial e no SAF-Frutíferas (SF) na camada de 0,09-0,21 m, porém, de forma geral, os tratamentos não indicaram degradação da estrutura do solo proveniente do manejo executado.

Tabela 2. Descrição do perfil cultural e da análise visual da estrutura do solo (VESS) com os valores do Score Visual (EV) para sistema agroflorestal no Planalto Catarinense.

Table 2. Cultural profile and visual analysis of soil structure (VESS) with the values of Visual Score (EV) to agroforestry system in Santa Catarina Plateau.

\begin{tabular}{|c|c|c|c|c|}
\hline$\frac{\text { Camadas }}{(\mathrm{m})}$ & Perfil Cultural & VESS $^{(1)}$ & Porosidade visível & $\mathbf{E V}^{(2)}$ \\
\hline \multicolumn{5}{|c|}{ Vegetação Nativa (VN) } \\
\hline $0,0-0,05$ & NAML $\mu$ & Qe1/Friável & Alta porosidade visível & 1,83 \\
\hline $0,05-0,25$ & NAML $\mu$ & Qe2/Intacto & Pouca porosidade visível & \\
\hline \multicolumn{5}{|c|}{ SAF-Agrícola (SA) } \\
\hline $0,0-0,09$ & $\mathrm{AML} \mu \Delta$ & Qe3/Firme & Baixa porosidade visível & 2,30 \\
\hline $0,09-0,21$ & $\mathrm{AML} \mu$ & Qe2/Intacto & Pouca porosidade visível & \\
\hline \multicolumn{5}{|c|}{ SAF-Frutíferas (SF) } \\
\hline $0,0-0,09$ & $\mathrm{AML} \mu$ & Qe2/Intacto & Pouca porosidade visível & 2,0 \\
\hline $0,09-0,21$ & $\operatorname{AML} \mu \Delta$ & Qe2/Intacto & Pouca porosidade visível & \\
\hline \multicolumn{5}{|c|}{ SAF-Erva-Mate (SE) } \\
\hline $0,0-0,05$ & $\mathrm{AML} \mu$ & Qe1/Friável & Alta porosidade visível & 1,83 \\
\hline $0,05-0,25$ & $\mathrm{AML} \mu$ & Qe2/Intacto & Pouca porosidade visível & \\
\hline
\end{tabular}

(1) VESS: avaliação visual da estrutura do solo; ${ }^{(2)}$ Cálculo de EV (Escore Visual) realizado de acordo com a equação: [nota Qe* camada avaliada $(\mathrm{cm}) /$ profundidade total $(\mathrm{cm}) \mid$ exemplo: $\left.\mathrm{VN}\left(1^{\star} 5 / 30\right)+\left(2^{\star} 25 / 30\right)\right]$ proposta por Ball et al. (2007). 
Os tratamentos VN e SAF-Erva Mate (SE) apresentaram em todo o perfil homogeneidade na distribuição e uniformidade no tamanho dos agregados, devido à não interferência do sistema de manejo do solo (NAM) no caso da VN e ao menor impacto na alteração da estrutura do solo no SE, ambos apresentaram porosidade visível (superfície rugosa dos agregados) além da presença de bioporos, o que pode favorecer a melhor circulação de água e ar pelo perfil do solo (Tavares et al., 1999; Lima et al., 2012).

Para o SF, na camada superficial, observou-se uma estrutura livre (L) e agregados não compactados, entretanto, na camada subsuperficial, os agregados apresentaram mais compactos $(\mu \Delta)$, ou seja, agregados com superfícies mais planas e lisas, formando ângulo reto em alguns casos.

No SA em maior profundidade, identificaram-se agregados de maior tamanho e mais rugosos, porém, na camada superficial $(0-0,09 \mathrm{~m})$, os agregados apresentaram-se mais firmes, com faces mais lisas, indicando, dessa forma, o início do processo compressivo $(\mu \Delta)$ provavelmente ocasionado pelo revolvimento do solo na ocasião do plantio (enxadas e cavadeiras), mesmo que localizado (Oliveira et al., 2004).

\subsection{Atributos físicos e análise visual da estrutura do solo}

O sistema agroflorestal mesmo em seu primeiro ano de cultivo já apresentou algumas alterações dos atributos físicos e hídricos do Cambissolo Húmico detectados tanto no tempo (anos de 2014, 2015) como entre os tratamentos.

A densidade do solo (Ds) apresentou diferenças significativas entre os tratamentos e o tempo na primeira camada de solo $(0-0,05 \mathrm{~m})$ com destaque para os tratamentos SE e SA que tiveram as maiores Ds $\left(>0,65 \mathrm{~g} \mathrm{~cm}^{-3}\right)$, especialmente para o ano de 2015 (Tabela 3), confirmando a alteração visual observada a campo para o $\mathrm{SA}(\mathrm{EV}=2,3$ e $\mathrm{L} \mu \Delta)$. Na segunda camada (0,05-0,20 m), houve diferenças significativas somente entre os tratamentos, porém com uma pequena redução na Ds em 2015.

Segundo Reichert et al. (2003), em solos com médio conteúdo de argila de $250 \mathrm{~g} \mathrm{~kg}^{-1}$, o valor crítico de Ds está entre 1,4 e 1,5 $\mathrm{Mg} \mathrm{m}^{-3}$, logo, neste estudo, que apresenta valores acima de $65 \%$ de argila, os solos apresentam menor Ds, o que favorece o desenvolvimento radicular das plantas, logo, um sistema agroflorestal que não utiliza o preparo primário e secundário de

Tabela 3. Atributos físicos do Cambissolo Húmico nas camadas de 0-0,05m e 0,05-0,20m de profundidade nos anos de 2014 e 2015 em um Sistema Agroflorestal no Planalto Catarinense.

Table 3. Physical properties of Inceptisol at 0-0,05m and $0.05-0.20 \mathrm{~m}$ layer in 2014 and 2015 years in agroforestry system in Santa Catarina Plateau.

\begin{tabular}{|c|c|c|c|c|c|c|c|c|}
\hline \multirow[b]{2}{*}{ Ano } & \multicolumn{2}{|c|}{$\frac{0-0,05 \mathrm{~m}}{\mathrm{Ds}^{(1)}}$} & \multicolumn{2}{|c|}{$\mathbf{P T}^{(2)}$} & \multicolumn{2}{|c|}{$\mathbf{M i}^{(3)}$} & \multicolumn{2}{|c|}{$\mathbf{M a}^{(4)}$} \\
\hline & 2014 & 2015 & 2014 & 2015 & 2014 & 2015 & 2014 & 2015 \\
\hline Tratamento & \multicolumn{2}{|c|}{.............Mg m ${ }^{-3} \ldots \ldots \ldots . . .}$. & \multicolumn{4}{|c|}{ 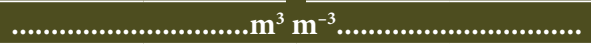 } & & \\
\hline SE & $0,67 \mathrm{aA}$ & $0,75 \mathrm{aA}$ & $0,50 \mathrm{aA}$ & $0,70 \mathrm{aA}$ & $0,35 \mathrm{aA}$ & $0,41 \mathrm{aA}$ & 0,15 aA & $0,29 \mathrm{aA}$ \\
\hline SA & $0,65 \mathrm{aA}$ & $0,78 \mathrm{aA}$ & $0,49 \mathrm{aA}$ & $0,67 \mathrm{aAB}$ & $0,36 \mathrm{aA}$ & $0,55 \mathrm{aA}$ & $0,13 \mathrm{bA}$ & $0,12 \mathrm{bAB}$ \\
\hline SF & $0,59 \mathrm{bA}$ & $0,55 \mathrm{bB}$ & $0,46 \mathrm{bA}$ & $0,50 \mathrm{bB}$ & $0,34 \mathrm{aA}$ & $0,40 \mathrm{aA}$ & $0,12 \mathrm{bA}$ & $0,10 \mathrm{bB}$ \\
\hline \multirow[t]{3}{*}{$\mathrm{VN}$} & $0,58 \mathrm{abA}$ & $0,72 \mathrm{abAB}$ & $0,45 \mathrm{abA}$ & $0,67 \mathrm{abAB}$ & $0,28 \mathrm{aA}$ & $0,51 \mathrm{aA}$ & $0,17 \mathrm{abA}$ & $0,15 \mathrm{abAB}$ \\
\hline & \multicolumn{2}{|c|}{$0,05-0,20 \mathrm{~m}$} & & & & & & \\
\hline & \multicolumn{2}{|c|}{ Ds } & \multicolumn{2}{|c|}{ PT } & \multicolumn{2}{|c|}{ Mi } & \multicolumn{2}{|c|}{ Ma } \\
\hline Ano & 2014 & 2015 & 2014 & 2015 & 2014 & 2015 & 2014 & 2015 \\
\hline Tratamento & \multicolumn{2}{|c|}{ 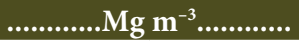 } & \multicolumn{4}{|c|}{ 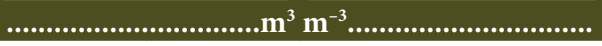 } & & \\
\hline SE & $0,88 \mathrm{aA}$ & $0,86 \mathrm{aA}$ & $0,59 \mathrm{aA}$ & $0,67 \mathrm{aA}$ & $0,38 \mathrm{aA}$ & $0,52 \mathrm{aA}$ & $0,21 \mathrm{aA}$ & $0,15 \mathrm{aA}$ \\
\hline SA & $0,88 \mathrm{aA}$ & $0,86 \mathrm{aA}$ & $0,59 \mathrm{aA}$ & $0,67 \mathrm{aAB}$ & $0,40 \mathrm{aA}$ & $0,49 \mathrm{aA}$ & $0,19 \mathrm{aA}$ & $0,18 \mathrm{aAB}$ \\
\hline SF & $0,78 \mathrm{aA}$ & $0,82 \mathrm{aB}$ & $0,55 \mathrm{aA}$ & $0,68 \mathrm{aB}$ & $0,37 \mathrm{aA}$ & $0,52 \mathrm{aA}$ & $0,17 \mathrm{aA}$ & $0,17 \mathrm{aB}$ \\
\hline VN & $0,81 \mathrm{aA}$ & $0,74 \mathrm{aAB}$ & $0,56 \mathrm{aA}$ & $0,68 \mathrm{aAB}$ & $0,39 \mathrm{aA}$ & $0,48 \mathrm{aA}$ & $0,24 \mathrm{aA}$ & $0,20 \mathrm{aAB}$ \\
\hline
\end{tabular}

SE: SAF-Erva Mate; SA: SAF-Agrícola; SF: SAF-Frutíferas e VN: Vegetação Nativa. ${ }^{(1)}$ Ds: densidade global; ${ }^{(2)}$ PT: porosidade total;

(3) Mi: microporosidade; ${ }^{(4)}$ Ma: Macroporosidade. Médias de tratamentos em cada profundidade, seguidas de letras minúsculas iguais na coluna e médias de tratamentos seguidas de letras maiúsculas iguais na linha não diferem pelo teste de Tukey a $5 \%$ de probabilidade. 
solo pode apresentar valores de Ds superiores nas camadas superficiais.

Os baixos valores encontrados de Ds refletem o caráter orgânico do Cambissolo. Solos com altos teores de matéria orgânica (Figura 1) ocupam a maior parte da fase sólida e, consequentemente, apresentarão Ds baixas (Loss et al., 2009; Luciano et al., 2012), entretanto, quando cultivados por longos períodos e em diferentes sistemas de manejo (convencional e plantio direto), esses valores podem ser elevados, devido à redução da matéria orgânica, dependendo do sistema de manejo utilizado (Bertol et al., 2015).

A porosidade total (PT) apresentou diferenças significativas no tempo, com valores maiores no segundo ano de cultivo do sistema, porém não houve diferença entre os tratamentos para a camada $0,05-0,20 \mathrm{~m}$. Kiehl (1979) observou que o intervalo de PT em solos arenosos deveria ser entre 0,35 e $0,50 \mathrm{~m}^{3} \mathrm{~m}^{-3} \mathrm{e}$ de 0,40 a $0,60 \mathrm{~m}^{-3} \mathrm{~m}^{-3}$ para solos argilosos, porém ainda afirmou que em solos com altos teores de matéria orgânica (Figura 1) a PT poderia apresentar entre 0,60 e $0,80 \mathrm{~m}^{3} \mathrm{~m}^{-3}$, o que explica os altos valores encontrados neste trabalho e observados em campo (L $\mu$ ) na maioria dos tratamentos (Tabela 2). Esses dados ainda são corroborados por Carvalho et al. (2004), que encontraram valores de PT acima de 60\% em Latossolo Vermelho argiloso sob sistema agroflorestal.

No caso da Vegetação Nativa, a elevada deposição da serapilheira favorece ainda a presença constante de minhocas responsáveis pela estruturação de galerias no solo, o que contribui com o aumento da macroporosidade e redução da densidade desse solo (Hariah et al., 2006).

Ao detalhar a porosidade desse solo em macro (Ma) e microporos (Mi), respectivamente, verificou-se que a Mi segue uma ordem crescente de um ano para outro nas duas camadas de solo em detrimento dos macroporos, exceto para o tratamento SE na camada superficial de solo, porém este parâmetro ainda apresentou valores acima do limite crítico ao crescimento radicular $\left(0,10 \mathrm{~cm}^{3} \mathrm{~cm}^{-3}\right)$ (Severiano et al., 2009).

Essa alteração na macroporosidade do solo na agrofloresta, especialmente no tratamento SE, pode ter ocorrido devido à presença de várias plantas de Erva-Mate (Ilex paraguariensis St. Hill.), que é uma cultura permanente, perene e típica da região, ou seja, seu sistema radicular pode adicionar matéria orgânica ao solo constantemente (Santos et al., 2009), além de plantas remanescentes que permanecem no sistema até o enriquecimento do SAF e que, segundo Salton et al. (2008) e Pedrotti \& Mello (2009), a atribuição dos sistemas de manejos conservacionistas, a exemplo do agroflorestal em estudo, pode induzir a permanente renovação e desenvolvimento de raízes, as quais vão originar um grande volume de bioporos, o que vem corroborar com o observado a campo onde o solo sob o tratamento SE obteve escore visual semelhante ao solo sob VN (Tabela 2).

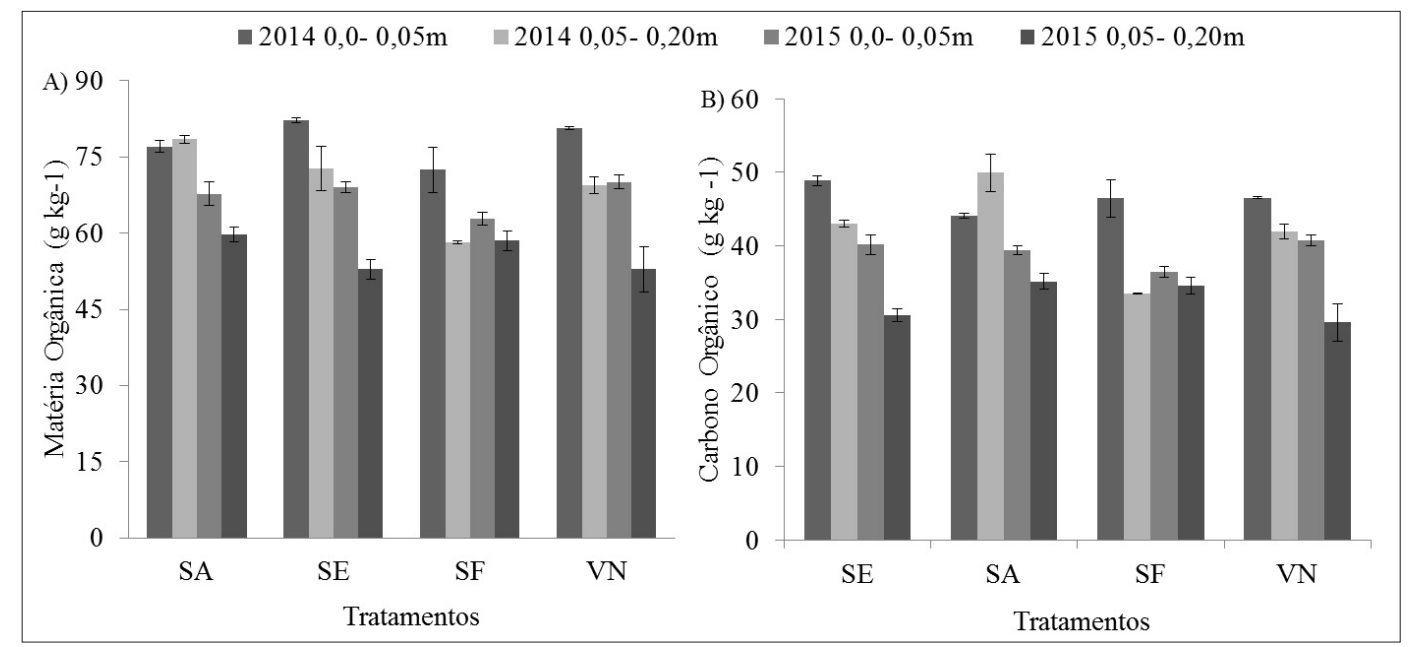

Figura 1. Conteúdo de matéria orgânica e carbono orgânico no solo em um sistema agroflorestal no Planalto Catarinense. SE: SAF-Erva Mate; SA: SAF-Agrícola; SF: SAF-Frutíferas e VN: Vegetação Nativa.

Figure 1. Organic matter content and soil organic carbon in agroforestry system in Santa Catarina Plateau. SE: SIF-Mate Herb; SA: SIF-Agricultural; SF: SIF-Fruit Trees and VN: Native Vegetation. 


\subsection{Atributos hídricos e análise visual da estrutura do solo}

De acordo com o balanço hídrico apresentado na Figura 2, pode-se observar que, ao longo dos monitoramentos, houve mais excessos hídricos do que déficit no sistema agroflorestal. Ressalta-se que o balanço hídrico para uma cultura varia no tempo e no espaço, ou seja, apresenta influência direta da profundidade de avaliação, como exigências das culturas e a armazenagem de água, além de indiretas: declividade da área, conteúdo de argila, infiltração de água e regime pluviométrico da região. Todos esses são agentes causadores de variabilidade da dinâmica da água no sistema solo-planta (Moreira et al., 2014).

Esses elevados valores de volume de água determinados no balanço hídrico são influenciados em grande parte pelo clima da região e, num segundo momento, pelo sistema agroflorestal (Carvalho et al., 2004; Loss et al., 2009).

O armazenamento da água no solo (Figura 3) apresentou os maiores valores no ano de 2015, especialmente na camada superficial de solo $(0-0,05 \mathrm{~m})$ e este foi superior aos valores de armazenamento referentes a 2014 para as duas camadas de solo em estudo. Em relação aos tratamentos agroflorestais em 2014, houve uma homogeneidade na quantidade de água armazenada e esta distribuição não foi observada em 2015, sendo que os maiores armazenamentos de água no solo ocorreram na $\mathrm{VN}$ e $\mathrm{SF}$, devido à presença de agregados mais compactos $(\mathrm{L} \mu \Delta)$ (Tabela 2), o que favorece o maior incremento em microporos no SAF na camada de 0,05-0,20m (Tabela 2).

Por outro lado, é importante ressaltar que 2015 foi o ano de ocorrência do fenômeno "EL NIÑO" que se caracteriza por elevadas temperaturas e chuvas acima da média, tendo maior influência no Sul do Brasil, na primavera (Figura 2), o que favorece o aumento das chuvas e tempestades severas, como é o caso da região em estudo.

Esse fenômeno climatológico atípico para a região também propiciou redução no conteúdo de matéria orgânica no solo, pela rápida decomposição (Figura 1), devido às temperaturas altas; o que, por outro lado, pode liberar substâncias orgânicas provenientes da decomposição de resíduos que favorecem em paralelo a formação de microagregados mais estáveis (Salton et al., 2008), o que estabelece uma relação direta e proporcional com os valores obtidos também da microporosidade (Loss et al., 2009).

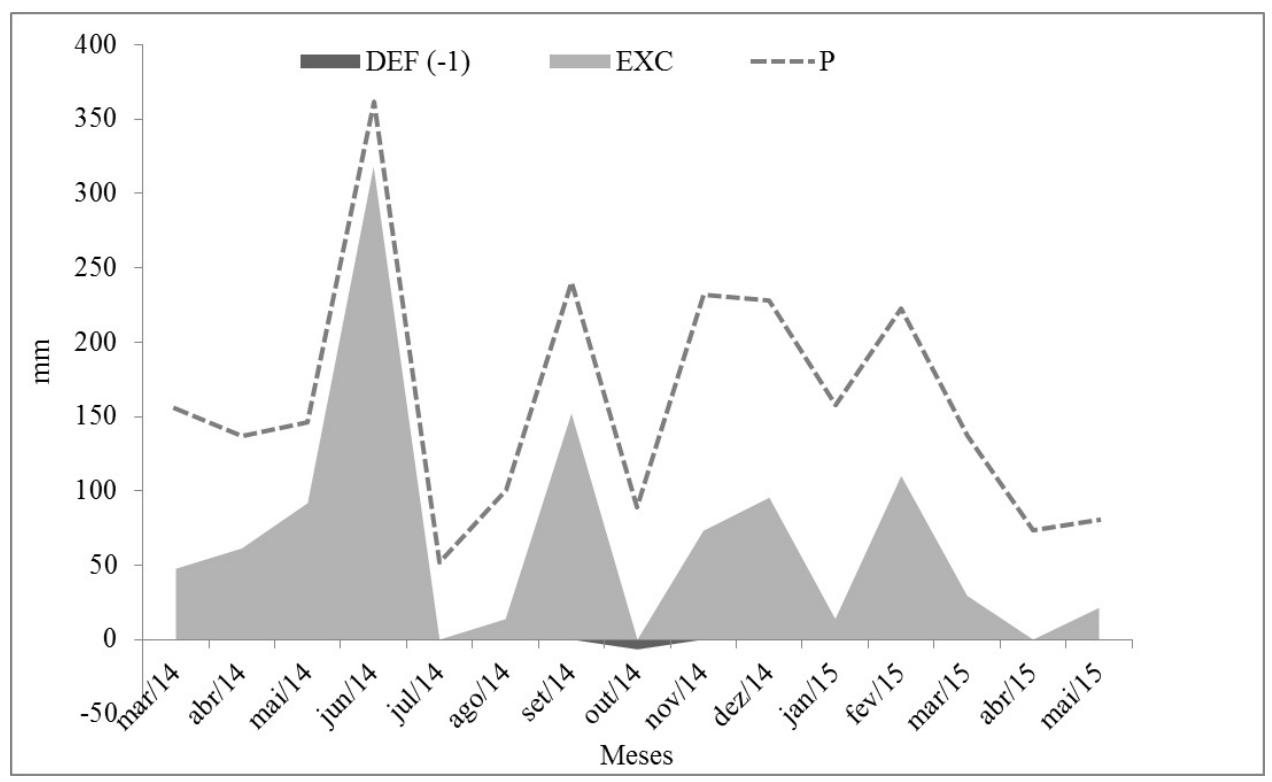

Figura 2. Balanço hídrico de um sistema agroflorestal no Planalto Catarinense. DEF(-1): Déficit hídrico; EXC: Excesso hídrico; P: Precipitação. Profundidade avaliada 0-0,20 m. Período condizente com as coletas.

Figure 2. Water balance of agroforestry system in Santa Catarina Plateau. DEF (1): Water deficit; EXC: water surplus; P: Precipitation. 0-0.20 m depth evaluated. Soil sampling period. 


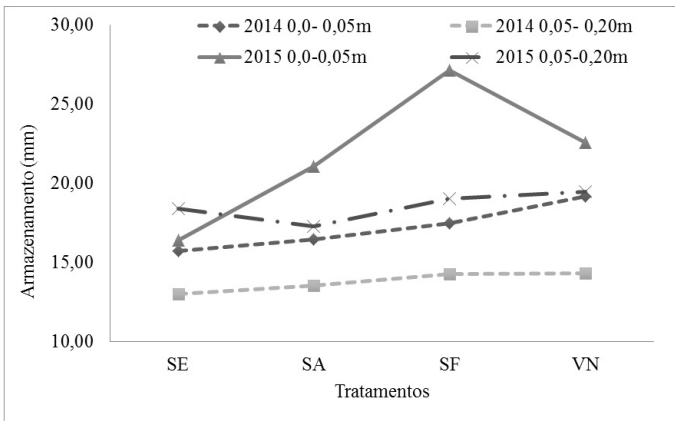

Figura 3. Armazenamento de água em um sistema agroflorestal no Planalto Catarinense. SE: SAF-Erva Mate; SA: SAF-Agrícola; SF: SAF-Frutíferas e VN: Vegetação Nativa.

Figure 3. Water storage in agroforestry system in Santa Catarina Plateau. SE: SIF-Mate Herb; SA: SIF- Agricultural; SF: SIF-Fruit Trees and VN: Native Vegetation.

\section{CONCLUSÃO}

A análise visual da estrutura do solo associada às avalições rotineiras dos atributos físicos e hídricos do solo permitiram identificar as alterações benéficas geradas pelo sistema agroflorestal no Cambissolo Húmico, já no primeiro ano de cultivo, especialmente com relação à capacidade do solo em armazenar e disponibilizar a água, o que torna o sistema agroflorestal uma forma viável e sustentável de produção agrícola e florestal além de promover a qualidade estrutural do solo.

\section{STATUS DA SUBMISSÃO}

Recebido: 13 set., 2016

Aceito: 3 nov., 2016

\section{AUTOR(ES) PARA CORRESPONDÊNCIA}

\section{Jânio dos Santos Barbosa}

Departamento de Agricultura, Biodiversidade e Florestas, Universidade Federal de Santa Catarina - UFSC, Rodovia Ulysses Gaboardi, Km 3, CEP 89520-000, Curitibanos, SC, Brazil e-mail: janio.jsb@gmail.com

\section{REFERENNCIAS}

Ball BC, Watson CA, Baddeley JA. Soil physical fertility, soil structure and rooting conditions after ploughinh organically managed grass/clover swards. Soil Use and Management 2007; 23(1): 20-27. http://dx.doi. org/10.1111/j.1475-2743.2006.00059.x.

Bertol I, Barbosa FT, Bertol C, Luciano RV. Water infiltration in two cultivated soils in Southern Brazil. Revista Brasileira de Ciencia do Solo 2015; 39(2): 573-588. http://dx.doi.or g/10.1590/01000683rbcs20140304.

Carvalho R, Goedert WJ, Armando MS. Atributos Físicos da qualidade de um solo sob sistema agroflorestal. Pesquisa Agropecuaria Brasileira 2004; 39(11): 1153-1155. http:// dx.doi.org/10.1590/S0100-204X2004001100015.

Empresa Brasileira de Pesquisa Agropecuária - EMBRAPA. Serviço nacional de pesquisa de solos: manual de métodos de análise de solo. 3. ed. Rio de Janeiro: Embrapa Solos; 2011. $230 \mathrm{p}$.

Giarola NFB, Silva AP, Tormena CA, Ball B, Rosa JA. Visual soil structure quality assessment on Oxisols under no-tillage system. Scientia Agricola 2010; 67(4): 497-482. http://dx.doi.org/10.1590/S0103-90162010000400016.

Hariah K, Sulistyani H, Suprayogo D, Widianto PP, Widodo RH, Noordwijk MV. Litter layer residence time in forest and coffe agroforestry systems in Sumberjaya, West Lampung. Forest Ecology and Management 2006; 224: 24-57.

Kiehl EJ. Manual de edafologia. São Paulo: Agronômica Ceres; 1979. 262 p.

Lima VMP, Oliveira GC, Serafim ME, Curi N, Evangelista AR. Intervalo hídrico ótimo como indicador de melhoria da qualidade estrutural de Latossolo degradado. Revista Brasileira de Ciencia do Solo 2012; 36(1): 71-78. http:// dx.doi.org/10.1590/S0100-06832012000100008.

Loss A, Pereira MG, Schultz N, Anjos LHC, Silva EMR. Atributos químicos e físicos de um Argissolo VermelhoAmarelo em sistema integrado de produção agroecológica. Pesquisa Agropecuaria Brasileira 2009; 44(1): 68-75. http:// dx.doi.org/10.1590/S0100-204X2009000100010.

Luciano RV, Albuquerque JA, Costa A, Batistella B, Warmling MT. Atributos físicos relacionados à compactação de solos sob vegetação nativa em região de altitude no Sul do Brasil. Revista Brasileira de Ciencia do Solo 2012; 36(6): 1733-1744. http://dx.doi.org/10.1590/S010006832012000600007 .

Moreira NB, Libardi PL, Salvador MMS, Sousa HHF. Análise espaço-temporal de componentes do balanço hídrico em um Latossolo. Revista Brasileira de Ciencia do Solo 2014; 38(1): 60-72. http://dx.doi.org/10.1590/ S0100-06832014000100006.

Oliveira GC, Dias MS Jr, Resck DVS, Curi N. Caracterização química e físico-hídrica de um Latossolo Vermelho após vinte anos de manejo e cultivo do solo. Revista Brasileira de Ciencia do Solo 2004; 28(2): 327-336. http://dx.doi. org/10.1590/S0100-06832004000200011. 
Pedrotti A, Mello AV Jr. Avanços em ciência do solo: a física do solo na produção agrícola e qualidade ambiental. Aracajú: Editora UFS; 2009. 212 p.

Pezarico CR, Vitorino ACT, Mercante FM, Daniel O. Indicadores de qualidade do solo em sistemas agroflorestais. Revista de Ciências Agrárias (Belém.) 2013; 56(1): 40-47. http://dx.doi.org/10.4322/rca.2013.004.

Reichert JM, Reinert DJ, Braida JÁ. Qualidade dos solos e sustentabilidade de sistemas agrícolas. Ciência e Ambiente 2003; 27: 29-48.

Reichert JM, Reinert DJ, Suzuki L, Horn R. Mecânica do solo. In: Van Lier QJ. Física do solo. Viçosa: Sociedade Brasileira de Ciência do Solo; 2010. p. 29-102.

Reichardt K, Timm LC. Solo, planta e atmosfera: conceitos, processos e aplicações. Barueri: Manole; 2004. 478 p.

Salton JC, Mielniczuk J, Bayer C, Boeni M, Conceição PC, Fabricio AC et al. Agregação e estabilidade de agregados do solo em sistemas agropecuários em Mato Grosso do Sul. Revista Brasileira de Ciencia do Solo 2008; 32(1): 11 21. http://dx.doi.org/10.1590/S0100-06832008000100002.

Santos RC, Woiciechowski T, Lombardi KC. Estudo preliminar dos efeitos do sombreamento de eucalipto (Eucalyptus dunnii MAIDEN) em plantio de erva-mate (Ilex paraguariensis ST. HILL.). Synergismus Scyentifica 2009; 4: 326-329.
Serafim ME, Oliveira GC, Lima JM, Silva BM, Zeviani WM, Lima VMP. Disponibilidade hídrica e distinção de ambientes para cultivo de cafeeiros. Revista Brasileira de Engenharia Agrícola e Ambiental 2013; 17(4): 362-370. http://dx.doi.org/10.1590/S1415-43662013000400002.

Severiano EC, Oliveira GC, Curi N, Dias MS Jr. Potencial de uso e qualidade estrutural de dois solos cultivados com cana-de-açúcar em goianésia (GO). Revista Brasileira de Ciencia do Solo 2009; 33(1): 159-168. http://dx.doi. org/10.1590/S0100-06832009000100017.

Silva DC, Silva MLN, Curi N, Oliveira AH, Souza F, Martins SG, Macedo RLG. Atributos do solo em sistemas agroflorestais, cultivo convencional e floresta nativa. Revista de Estudos Ambientais 2011; 13: 77-86.

Tavares J Fo, Ralisch R, Guimarães MF, Medina CC, Balbino LC, Neves CSVJ. Método do perfil cultural para avaliação do estado físico de solos em condições tropicais. Revista Brasileira de Ciencia do Solo 1999; 23(2): 393-399. http://dx.doi.org/10.1590/S0100-06831999000200022.

Thornthwaite CW, Mather JR. The water balance. Centerton: Drexel Institute of Technology; 1955. 104 p. Publications in Climatology, vol. VIII, n. 1.

Wrege MS, Steinmetz S, Reisser C Jr, Almeida IR. Atlas climático da Região Sul do Brasil. Pelotas: Embrapa Clima Temperado; Colombo: Embrapa Florestas; 2011. 333 p. 